\title{
Elation switching in real parallelisms
}

\author{
Esteban Diaz Norman L. Johnson \\ Alessandro Montinaro*
}

full screen

close quit

\begin{abstract}
Switching techniques are developed that produce a variety of new parallelisms in $\mathrm{PG}(3, K)$, where $K$ is a infinite field. When $K$ is the field of real numbers, $2^{\chi_{0}}$ mutually non-isomorphic parallelisms are constructed.
\end{abstract}

Keywords : parallelisms, deficiency one, transitive groups

MSC 2000: primary: 51E23; secondary: 51A40

\section{Introduction}

A parallelism of a projective space $\mathrm{PG}(3, F)$, where $F$ is a skew field, is an equivalence relation on the set of lines satisfying the Euclidean parallel postulate. This concept originated with Clifford's work [3] in 1873, where there are two parallelisms. Indeed, there are characterizations of parallelisms admitting what are called left and right parallelisms and having certain other properties (see, e.g. the work of Karzel [11] and Karzel and Kroll [12]). More recently, there are a variety of parallelisms constructed by Betten and Riesinger over PG $(3, \mathcal{R})$, where $\mathcal{R}$ is the field of real numbers (see [1]). Indeed, there are also a variety of real parallelisms constructed by the second author and R. Pomareda in [10]. It might be mentioned that the equivalence classes of a parallelism, called spreads, define affine translation planes in the associated four dimensional vector space over $F$ and analysis of affine geometry then provides a strong technical device for the study of parallelisms.

\footnotetext{
*The ideas for this paper were conceived when the third author was visiting the University of Iowa during the Spring semester of 2007. The authors are grateful to the university for support on this research.
} 




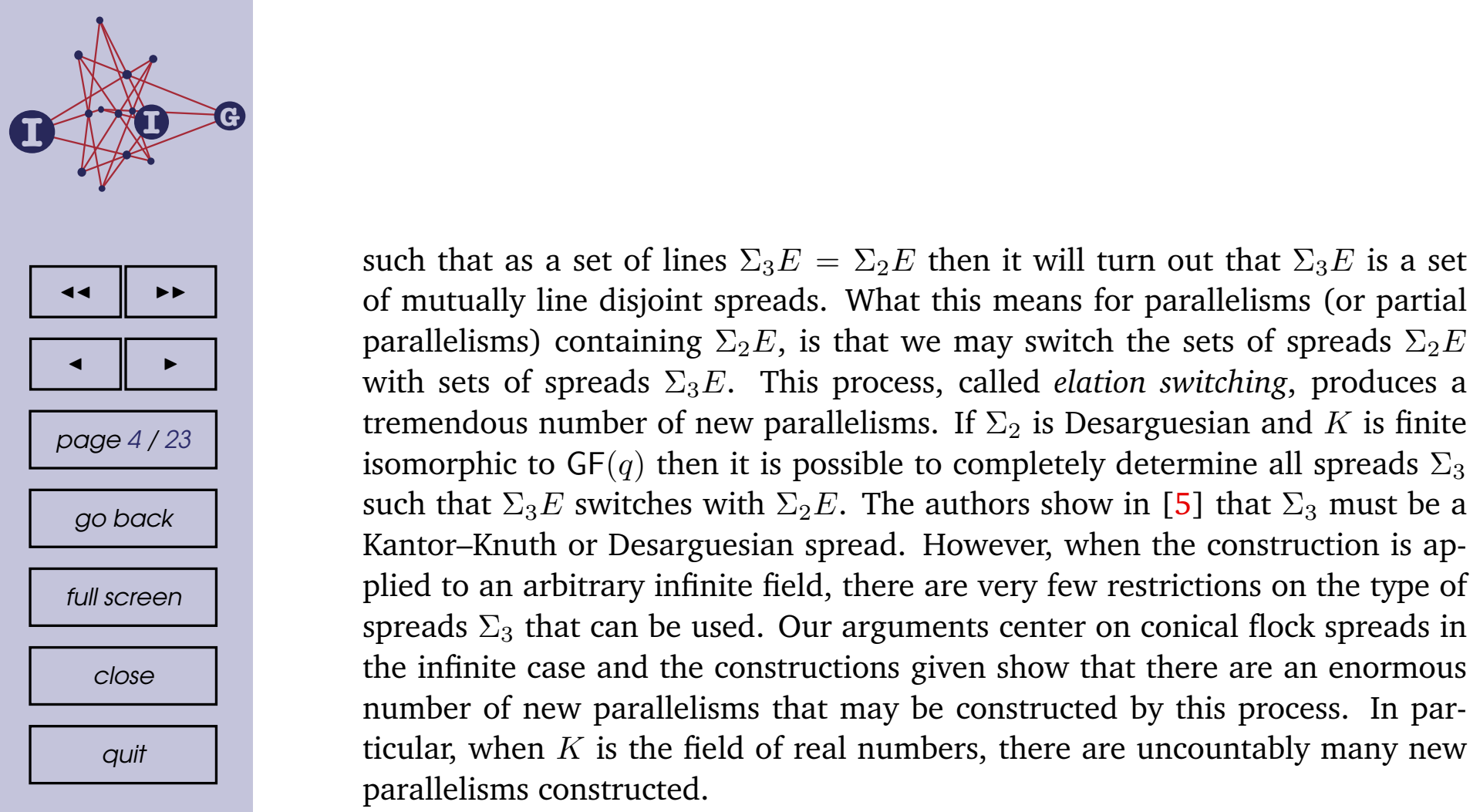

\section{Elation switching}

As suggested previously, the application of this construction technique mentioned in the introduction has been applied most successfully when the spreads other than the Pappian spread are derived conical flock spreads and when the group contains a large normal subgroup that is a central collineation group. (By conical flock spreads, we intend to mean those spreads that correspond to flocks of quadratic cones.) The reader is directed to the Handbook [8] for the precise definitions and additional background.

Actually, there is a classification by procedure of such parallelisms.

Theorem 2.1 (see Johnson [6]). Let $\mathcal{P}$ be a parallelism in $\mathrm{PG}(3, K)$, for $K$ a field, that admits a Pappian spread $\Sigma$ and a collineation group $G^{-}$fixing a line $\ell$ of $\Sigma$ that acts transitively on the remaining spreads of $\mathcal{P}$.

(1) If $K$ is finite and if $G^{-}$contains the full elation group with axis $\ell$ then the spreads of $\mathcal{P}-\{\Sigma\}$ are derived conical flock spreads.

(2) If $G^{-}$contains the full elation group with axis $\ell$ and, for $\rho$ a spread of $\mathcal{P}-\{\Sigma\}$, $G_{\rho}^{-}$contains a non-trivial homology (i.e. homology in $\Sigma$ ) then the spreads of $\mathcal{P}-\{\Sigma\}$ are derived conical flock spreads.

In a previous article on the above constructed over infinite fields, the second author constructed a variety of parallelisms over the reals (when $K$ is the field of real numbers, $K=\mathcal{R}$.

The main idea is as follows. Let a Pappian spread $\Sigma_{1}$ defined as follows: 


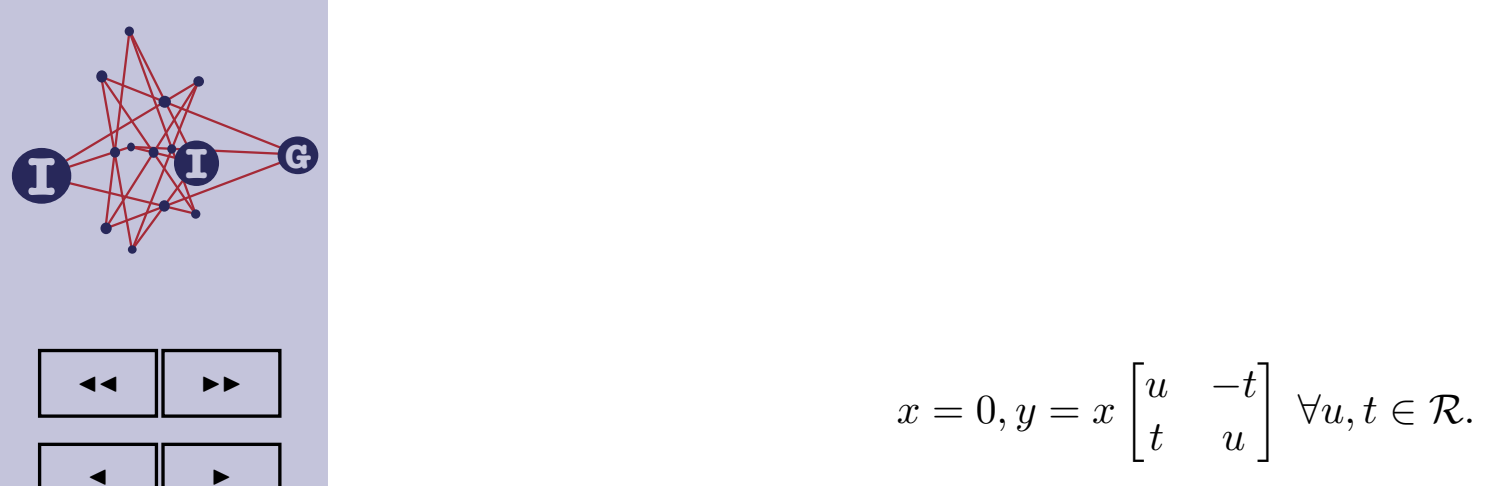

We let $\Sigma_{2}$ be a spread in $\mathrm{PG}(3, \mathcal{R})$, defined by a function $f$ :

$$
x=0, y=x\left[\begin{array}{cc}
u & -f(t) \\
t & u
\end{array}\right] \forall u, t \in \mathcal{R}
$$

where $f$ is a function such that $f(t)=t$ implies that $t=0$ and $f(0)=0$.

Thus, if a spread exists then the two spreads $\Sigma_{1}$ and $\Sigma_{2}$ share exactly the regulus $\mathcal{D}$ with partial spread:

$$
x=0, y=x\left[\begin{array}{ll}
u & 0 \\
0 & u
\end{array}\right] \forall u \in \mathcal{R} .
$$

The following lemma of Johnson and Pomareda connects at least one of the groups $G$ that we use for our construction.

Lemma 2.2 (Johnson and Pomareda [10]). Let $f$ be any continuous strictly increasing function on the field of real numbers such that $\lim _{x \rightarrow \pm \infty} f(t)= \pm \infty$.

(1) Then $\Sigma_{2}$ is a spread.

(2) Let $G^{-}=E H^{-}$where $H^{-}$denotes the homology group of $\Sigma_{1}$ (or rather the associated affine plane) whose elements are given by

$$
\left\langle\left[\begin{array}{cccc}
u & -t & 0 & 0 \\
t & u & 0 & 0 \\
0 & 0 & 1 & 0 \\
0 & 0 & 0 & 1
\end{array}\right] ; u^{2}+t^{2}=1\right\rangle
$$

and where $E$ denotes the full elation group with axis $x=0$.

(3) Then $G^{-}$is transitive on the set of reguli of $\Sigma_{1}$ that share $x=0$.

Then for conditions that such spreads produce parallelisms along the same lines as when the second chosen spread is Pappian, we mention the following result:

Theorem 2.3 (Johnson and Pomareda [10]). Under the above assumptions, assume also that $f$ is symmetric with respect to the origin in the real Euclidean 2-space and $f\left(t_{o}+r\right)=f\left(t_{o}\right)+r$ for some $t_{o}$ and $r$ in the reals implies that $r=0$.

(1) Then

$$
\Sigma_{1} \cup \Sigma_{2}^{*} g ; g \in G^{-}
$$

is a partial parallelism $\mathcal{P}_{f}$ in $\mathrm{PG}(3, \mathcal{R})$, where $\Sigma_{2}^{*}$ denotes the derived spread of $\Sigma_{2}$ by derivation of $\mathcal{D}$. 


for all $x_{1}, x_{2}, y, y_{2} \in K$. If $x_{1}=0$, clearly, there is a unique solution for $(u, t)$ and hence a unique $y=x\left[\begin{array}{cc}u & f(t) \\ t & u\end{array}\right]$ that covers the given point.

If $x_{2}=0$ then $x_{1} f(t)=y_{2}$ provided $f$ is bijective and again there is a unique solution for $(u, t)$.

So, assume that $x_{1} x_{2} \neq 0$. Then,

go back

full screen

$$
\begin{aligned}
u+z t & =y_{1}^{*}, \\
u+z^{-1} f(t) & =y_{2}^{*},
\end{aligned}
$$

where $z=x_{2} / x_{1}, y_{1}^{*}=y_{1} / x_{1}$ and $y_{2}^{*}=y_{2} / x_{2}$. Note that $y_{1}^{*}$ and $y_{2}^{*}$ and $z$ are then completely independent. Thus,

$$
z t-z^{-1} f(t)=\frac{z^{2} t-f(t)}{z} .
$$

Since $f(t)-z^{2} t$ is bijective for all elements $z^{2}$, we have a unique solution for $t$, which then produces a unique solution $(u, t)$. This completes the proof of the theorem.

Now we shall be interested in spreads of the above form that share precisely a regulus with a Pappian spread

$$
\left\{x=0, y=x\left[\begin{array}{cc}
u & \gamma_{1} t \\
t & u
\end{array}\right] \forall u, t \in K\right\} \text {, where } \gamma_{1} \text { is a non-square in } K,
$$

and such that the full elation subgroup of $E$ that acts as a collineation group of the spread in question is

(i) $E^{-}=\left\langle\left[\begin{array}{llll}1 & 0 & u & 0 \\ 0 & 1 & 0 & u \\ 0 & 0 & 1 & 0 \\ 0 & 0 & 0 & 1\end{array}\right] ; u \in K\right\rangle$, where

(ii) the full elation of $\Sigma_{1}$ with axis $x=0$ is

$$
E=\left\langle\left[\begin{array}{cccc}
1 & 0 & u & \gamma_{1} t \\
0 & 1 & t & u \\
0 & 0 & 1 & 0 \\
0 & 0 & 0 & 1
\end{array}\right] ; u, t \in K\right\rangle, \text { and }
$$

(iii) such that for any elation $e \in E-E^{-}$then $\Sigma_{f} e \cap \Sigma_{f}$ is empty.

The following proposition is essentially immediate and is left to the reader to verify. 
Proposition 2.6. A spread

$$
\Sigma_{f}=\left\{x=0, y=x\left[\begin{array}{cc}
u & f(t) \\
t & u
\end{array}\right] \forall u, t \in K\right\}
$$

shares exactly the regulus

$$
R_{0}=\left\{x=0, y=x\left[\begin{array}{ll}
u & 0 \\
0 & u
\end{array}\right] \forall u \in K\right\}
$$

with the Pappian spread $\Sigma_{1}$ if and only if

$$
f(t)-\gamma_{1} t=0
$$

implies $t=0$.

Note that in the finite case, $f$ is forced to be additive and so the above condition implies that the function $f-\gamma_{1}$ is bijective (injective will suffice in the finite setting). This injective condition turns out to be an important condition for elation switching.

Definition 2.7. If the function $g$ defined by $g(t)=f(t)-\gamma_{1} t$ is injective, we shall say that the function $f$ has the regulus property.

If the full elation group of a spread $\Sigma_{f}$ of $E$ is $E^{-}$, and for $e \in E-E^{-}$then $\Sigma_{f} e \cap \Sigma_{f}$ is empty, we shall say that the spread has the regulus-inducing property.

For example, if $f(t)=\gamma_{2} t$ and $\gamma_{2} \neq \gamma_{1}$ then $f$ will turn out to have the regulus-inducing property. For the automorphism type function $f(t)=\gamma_{2} t^{\sigma}$ we need $\gamma_{2} t_{0}^{\sigma}=\gamma_{1} t_{0}$ for some $t_{0}$ if and only if $t=0$.

More generally, we have the following description of spreads that have the regulus-inducing property.

Proposition 2.8. A spread

$$
\Sigma_{f}=\left\{x=0, y=x\left[\begin{array}{cc}
u & f(t) \\
t & u
\end{array}\right] \forall u, t \in K\right\},
$$

has the regulus-inducing property if and only if for $t_{0}, r \in K$

$$
f\left(t_{0}+r\right)=f\left(t_{0}\right)+\gamma_{1} r
$$

implies $r=0$. 



\section{Main theorem on elation switching}

Theorem 3.1. Let $\Sigma_{0}$ denote a Pappian spread in $\mathrm{PG}(3, K)$, where $K$ is a field:

$$
\left\{x=0, y=x\left[\begin{array}{cc}
u & \gamma_{1} t \\
t & u
\end{array}\right] \forall u, t \in K\right\} \text {, where } \gamma_{1} \text { is a non-square in } K \text {. }
$$

go back

full screen

close

quit

Let $E$ denote the full elation group of $\Sigma_{0}$ with axis $x=0$ :

$$
E=\left\langle\left[\begin{array}{cccc}
1 & 0 & u & \gamma_{1} t \\
0 & 1 & t & u \\
0 & 0 & 1 & 0 \\
0 & 0 & 0 & 1
\end{array}\right] ; u, t \in K\right\rangle
$$

Assume that $\Sigma_{i}$ is a spread in $\mathrm{PG}(3, K)$ of the following form:

$$
\Sigma_{i}=\left\{x=0, y=x\left[\begin{array}{cc}
u & f_{i}(t) \\
t & u
\end{array}\right] \forall u, t \in K\right\}, \text { for } i=2,3,
$$

where $f_{i}$ is a function $K \rightarrow K$ and such that both spreads admit the switching property.

Then $\Sigma_{2} E$ switches with $\Sigma_{3} E$.

Proof. Note that $\Sigma_{2} E=\Sigma_{3} E$ if and only if $\Sigma_{2}$ is in $\Sigma_{3} E$ and $\Sigma_{3}$ is in $\Sigma_{2} E$. Note that

$$
y=x\left[\begin{array}{cc}
u & f_{i}(t) \\
t & u
\end{array}\right]
$$

maps to

$$
y=x\left\{\left[\begin{array}{cc}
u & f_{i}(t) \\
t & u
\end{array}\right]+\left[\begin{array}{cc}
u^{*} & \gamma_{1} t^{*} \\
t^{*} & u^{*}
\end{array}\right]\right\}, \forall u^{*}, t^{*} \in K
$$

by $E$ and note that $E$ fixes $x=0$ pointwise.

So consider, for $j \neq i$,

$$
\left[\begin{array}{cc}
u & f_{i}(t) \\
t & u
\end{array}\right]=\left[\begin{array}{cc}
u & f_{j}(s) \\
s & u
\end{array}\right]+\left[\begin{array}{cc}
0 & \gamma_{1}(t-s) \\
(t-s) & 0
\end{array}\right] .
$$

Then

$$
f_{i}(t)=f_{j}(s)+\gamma_{1}(t-s)
$$

if and only if

$$
f_{j}(t)-\gamma_{1} t=f_{j}(s)-\gamma_{1} s
$$

Therefore, given $t$ in $K$, then there exists a unique $s$ in $K$ such that

$$
f_{j}(t)-\gamma_{1} t=f_{i}(s)-\gamma_{1} s,
$$




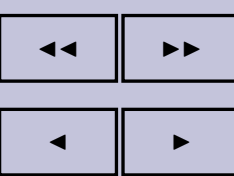

page $13 / 23$

go back

full screen

close

quit

where $\gamma_{2}$ is a negative element in $K, \gamma_{2} \neq \gamma_{1}$. Let $R_{0}$ denote the common regulus $\left\{x=0, y=x\left[\begin{array}{ll}u & 0 \\ 0 & u\end{array}\right] \forall u \in K\right\}$.

Theorem 4.1. $\Sigma_{1} \cup \Sigma_{2}^{*} E H$ is a parallelism in $\mathrm{PG}(3, K)$. Furthermore,

$$
E^{-}\left\langle\left[\begin{array}{cccc}
1 & 0 & 0 & 0 \\
0 & 1 & 0 & 0 \\
0 & 0 & -1 & 0 \\
0 & 0 & 0 & -1
\end{array}\right]\right\rangle
$$

is the subgroup of $E H$ that leaves $\Sigma_{2}$ invariant, where

$$
E^{-}=\left\langle\left[\begin{array}{cccc}
1 & 0 & u & 0 \\
0 & 1 & 0 & u \\
0 & 0 & 1 & 0 \\
0 & 0 & 0 & 1
\end{array}\right] ; u \in K\right\rangle
$$

Proof. First of all we claim that $E H$ is transitive on the set of reguli of $\Sigma_{1}$ that share $x=0$. We note that $E$ is transitive on the components of $\Sigma_{1}-\{x=0\}$. So the question then is $H$ transitive on the reguli that share $x=0$ and $y=0$. Any such regulus has the following form:

$$
R_{t}=\left\{x=0, y=0, y=x\left[\begin{array}{cc}
u & \gamma_{1} t \\
t & u
\end{array}\right] ; u \in K-\{0\}\right\} .
$$

So, the question is whether $R_{0}$ can be mapped into $R_{t}$ by an element of $H$. Hence, given $y=x\left[\begin{array}{cc}0 & \gamma_{1} t \\ t & 0\end{array}\right]$, we need to find an element $y=x\left[\begin{array}{ll}v & 0 \\ 0 & v\end{array}\right], v$ not zero, such that

$$
\left[\begin{array}{ll}
v & 0 \\
0 & v
\end{array}\right] w=\left[\begin{array}{cc}
u & \gamma_{1} t \\
t & u
\end{array}\right]
$$

for some $u \in K$.

Note that since clearly the reguli sharing $x=0, y=0$ are permuted by $H$, it just takes one appropriate image to establish that $R_{0}$ is mapped onto $R_{t}$, as any three distinct components generate a unique regulus of $\Sigma_{1}$. Since $w$ commutes with $\left[\begin{array}{ll}v & 0 \\ 0 & v\end{array}\right]$, we only need to show that for some element $\left[\begin{array}{cc}u & \gamma_{1} t \\ t & u\end{array}\right]$, for $t$ not zero, there exist elements $\left[\begin{array}{ll}v & 0 \\ 0 & v\end{array}\right]$ and $\left[\begin{array}{cc}u^{*} & \gamma_{1} t^{*} \\ t^{*} & u^{*}\end{array}\right]$, where $u^{* 2}-\gamma_{1} t^{* 2}=1$ such that

$$
\left[\begin{array}{cc}
v & 0 \\
0 & v
\end{array}\right]\left[\begin{array}{cc}
u^{*} & \gamma_{1} t^{*} \\
t^{*} & u^{*}
\end{array}\right]=\left[\begin{array}{cc}
u & \gamma_{1} t \\
t & u
\end{array}\right]
$$








\section{Examples}

Let $F$ be any subfield of the field of real numbers and let $F^{C}=K$ denote the field of constructible numbers from $F$. If we let $\gamma_{1}=-1$, choose any function $f_{i}$ where $f_{i}(t)=\gamma_{i} t$, for $\gamma_{i} \neq-1$. Then it is clear that the following define bijective functions:

$$
\begin{aligned}
\rho_{i, z}(t) & =f_{i}(t)-z^{2} t \text { and } \\
\phi_{i}(t) & =f_{i}(t)-\gamma_{1} t .
\end{aligned}
$$

Hence, we have the following result.

Theorem 6.1. Let $K=F^{C}$, a field of constructible numbers from a subfield $F$ of the field of real numbers. Let $\Sigma_{1}$ denote the Pappian spread

$$
\Sigma_{1}=\left\{x=0, y=x\left[\begin{array}{cc}
u & -t \\
t & u
\end{array}\right] \forall u, t \in K\right\}
$$

and let

$$
\begin{gathered}
E=\left\langle\left[\begin{array}{cccc}
1 & 0 & u & \gamma_{1} t \\
0 & 1 & t & u \\
0 & 0 & 1 & 0 \\
0 & 0 & 0 & 1
\end{array}\right] ; u, t \in K\right\rangle, \\
H=\left\langle\left[\begin{array}{cc}
1 & 0 \\
0 & w
\end{array}\right] ; w=\left[\begin{array}{cc}
u & \gamma_{1} t \\
t & u
\end{array}\right] ; u^{2}-\gamma_{1} t^{2}=1\right\rangle .
\end{gathered}
$$

Let $\left\{h_{i} ; i \in \lambda\right\}$ be a coset representation for

$$
H^{-}=\left\langle\left[\begin{array}{cccc}
1 & 0 & 0 & 0 \\
0 & 1 & 0 & 0 \\
0 & 0 & -1 & 0 \\
0 & 0 & 0 & -1
\end{array}\right]\right\rangle
$$

in $H$. For each $i \in \lambda$, choose a negative number $\gamma_{i}$ in $K$ such that $\gamma_{i} \neq-1$ and finally let

$$
\Sigma_{i}=\left\{x=0, y=x\left[\begin{array}{cc}
u & \gamma_{i} t \\
t & u
\end{array}\right] \forall u, t \in K\right\}
$$

Then

$$
\Sigma_{1} \cup_{i \in \lambda} \Sigma_{i}^{*} E h_{i}
$$

is a parallelism in $\mathrm{PG}(3, K)$. 
Remark 6.2. Let $\sigma_{i}$ be an automorphism of $K$. We consider functions $f_{i}$ such that $f_{i}(t)=\gamma_{i} t^{\sigma_{i}}$, where $\gamma_{i}$ is a negative number. In order to obtain parallelisms in a manner similar to that of the previous theorem, we need to check that the following define bijective functions:

$$
\begin{aligned}
\rho_{i, z}(t) & =\gamma_{i} t^{\sigma}-z^{2} t \text { and } \\
\phi_{i}(t) & =\gamma_{i} t^{\sigma}-\gamma_{1} t .
\end{aligned}
$$

Note that first set of functions $\rho_{i, z}$ are always

$$
f_{i}(t)-z^{2} t=\gamma_{i} t^{\sigma_{i}}-z^{2} t=0
$$

if and only if $t=0$ since $\gamma_{i}<0$. Since the function is additive, we see $\rho_{i, z}$ is injective.

In general, the surjectivity of $\rho_{i, z}$ is not always guaranteed.

\subsection{Examples over the reals}

Let

$$
f(t)=\left\{\begin{array}{ll}
\gamma_{1} t-a^{t}+1, & t \geq 0 \\
\gamma_{1} t+b^{-t}-1, & t<0
\end{array}\right\}, a, b \text { both }>1 .
$$

We see that $f(0)=0, f$ is continuous at all elements $t$ of the reals and consider $f(t)-z^{2} t$.

$$
f(t)-z^{2} t=\left\{\begin{array}{ll}
\gamma_{1} t-a^{t}+1-z^{2} t, & t \geq 0 \\
\gamma_{1} t+b^{-t}-1-z^{2} t, & t<0
\end{array}\right\}, a, b \text { both }>1
$$

Note that

$$
\lim _{t \rightarrow \pm \infty}-f(t)= \pm \infty
$$

so that $f(t)-z^{2} t$ is continuous and hence surjective. We note that the $\lim _{t \rightarrow 0} f(t)=$ 0 and $f^{\prime}(t)$ for $t$ non-zero is

$$
f^{\prime}(t)-z^{2}=\left\{\begin{array}{ll}
\gamma_{1}-a^{t} \ln a-z^{2}, & t \geq 0 \\
-1-b^{-t} \ln b-z^{2}, & t<0
\end{array}\right\}, a, b \text { both }>1
$$

which is never 0 . Hence, $f(t)-z^{2} t$ is bijective for each $z$. Now $f(t)-\gamma_{1} t$

$$
f(t)-\gamma_{1} t=\left\{\begin{array}{cc}
-a^{t}+1, & t \geq 0 \\
b^{-t}-1, & t<0
\end{array}\right\}, a, b \text { both }>1,
$$





\section{The derive-underive parallelisms}

We may now construct parallelisms from any parallelism of the type

$$
\Sigma_{1} \cup_{i \in \lambda} \Sigma_{f_{i}}^{*} E h_{i} .
$$

as follows: Choose an element $e h_{j}$ of $E h_{j}$, for some $j \in \lambda$. There is a regulus $R_{e h_{j}}$ of $\Sigma_{1}$ that is derived in $\Sigma_{f_{j}} e h_{j}$ to construct $\Sigma_{f_{j}}^{*} e h_{j}$. Derive $R_{e h_{j}}$ in $\Sigma_{1}$ to construct the Hall plane $\Sigma_{1}^{R_{e h_{j}}^{*}}$ and underive $R_{e h_{j}}^{*}$ in $\Sigma_{f_{j}}^{*} e h_{j}$ to construct $\Sigma_{f_{j}} e h_{j}$.

Theorem 7.1.

$$
\Sigma_{1}^{R_{e h_{j}}^{*}} \cup_{i \in \lambda-\{j\}} \Sigma_{f_{i}}^{*} E h_{i} \cup_{g \in E-\{e\}} \Sigma_{f_{j}}^{*} E h_{j} \cup \Sigma_{f_{j}} e h_{j}
$$

is a parallelism.

\section{The variety of parallelisms}

We note that although our original parallelism admits the group $E H$, the collineation group of certain of the constructed parallelisms can be made so that only $E$ is a collineation group. Furthermore, certain of the derive-underive parallelisms can be found that do not admit a non-trivial collineation.

We note that to construct parallelisms over the reals of the type here considered, it is sufficient to constructs functions $f_{i}$ with the conditions given in Theorem 5.1. We have also constructed $2^{\chi_{0}}$ different functions $f_{i}$ and therefore, we have also constructed $2^{\chi_{0}}$ distinct parallelisms of the type

$$
\Sigma_{1} \cup_{i \in \lambda} \Sigma_{f_{i}}^{*} E h_{i} .
$$

Any isomorphism between two parallelisms

$$
\Sigma_{1} \cup_{i \in \lambda} \Sigma_{f_{i}}^{*} E h_{i}
$$

and

$$
\Sigma_{1} \cup_{i \in \lambda} \Sigma_{g_{i}}^{*} E h_{i}
$$

of this type necessarily is a collineation group of $\Sigma_{1}$, the Pappian plane over the field of complex numbers (assuming that none of the derived conical flock spreads are Pappian). Since our parallelisms admit $E$, it follows that any isomorphism must be a collineation of $\Sigma_{1}$ that leaves $x=0, y=0$ invariant and must permute the set of reguli of $\Sigma_{1}$ sharing $x=0, y=0$ and so must permute the $\Sigma_{f_{i}}^{*} h_{i}$. Hence, there is a collineation of $\Sigma_{1}$ that would map a function $f_{i}$ 

